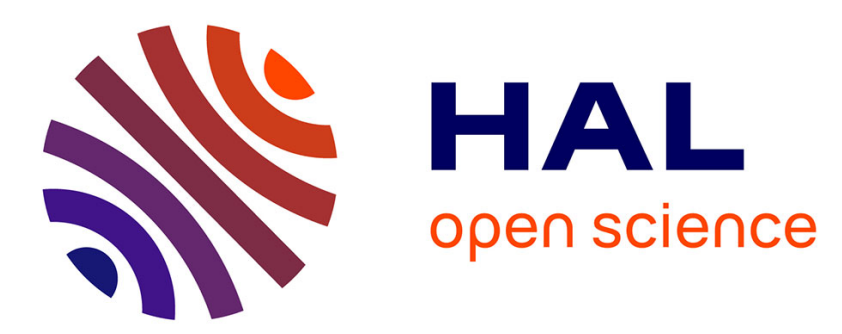

\title{
Design of thermoelastic multi-material structures with graded interfaces using topology optimization
}

Alexis Faure, Georgios Michailidis, Guillaume Parry, Natasha Vermaak, Rafael Estevez

\section{- To cite this version:}

Alexis Faure, Georgios Michailidis, Guillaume Parry, Natasha Vermaak, Rafael Estevez. Design of thermoelastic multi-material structures with graded interfaces using topology optimization. 2016. hal-01383393

\section{HAL Id: hal-01383393 \\ https://hal.science/hal-01383393}

Preprint submitted on 19 Oct 2016

HAL is a multi-disciplinary open access archive for the deposit and dissemination of scientific research documents, whether they are published or not. The documents may come from teaching and research institutions in France or abroad, or from public or private research centers.
L'archive ouverte pluridisciplinaire HAL, est destinée au dépôt et à la diffusion de documents scientifiques de niveau recherche, publiés ou non, émanant des établissements d'enseignement et de recherche français ou étrangers, des laboratoires publics ou privés. 


\title{
Design of thermoelastic multi-material structures with graded interfaces using topology optimization
}

\author{
Faure A. ${ }^{1}$, Michailidis G. ${ }^{1}$, Parry G. ${ }^{1}$, Vermaak N. ${ }^{3}$, Estevez R. ${ }^{2}$ \\ Received: - / Accepted: - \\ ${ }^{1}$ Université Grenoble Alpes, 1130 rue de la Piscine B.P. 75, 38402 St.Martin d'Héres, Cedex, \\ France \\ ${ }^{2}$ UMR 5266 CNRS / INPG / UJF, 1130 rue de la Piscine B.P. 75, 38402 St. Martin d'Héres, \\ Cedex,France \\ ${ }^{3}$ Mechanical Engineering and Mechanics, Lehigh University, 19 Memorial DriveWest, Bethlehem, \\ PA 18015-3085, USA
}

\begin{abstract}
A level set based shape and topology optimization framework is used to study the effect of graded interfaces in the optimization process of micro-architectured multi-materials. In contrast to previous studies interfaces are considered as smooth transition between phases instead of a sharp delimitation between two phases. A study on extreme thermoelastic properties of 2D isotropic composites is achieved and optimal design are presented. The study shows how taking into account smooth interfaces can influence the optimal design of these materials.
\end{abstract}




\section{Introduction}

Architectured materials are a new class of materials whose properties are governed not only by their constitutive phases volume fraction but their properties are also dependant by the geometry. In this case, architecture refers to the materials organization on a scale that is between the material microstructure and the macroscopic shape (Ashby, 2013; Ashby and Brechet, 2003; Brechet and Embury, 2013; Torquato, 2010). Beyond the chemical composition or the microstructure, architecture can also be regarded as a parameter to control the material's design to reach target effective properties. This new degree of freedom allows for properties that are unattainable with assemblies of bulk materials.

Recent improvements in manufacturing methods also offer new possibilities for proceeding architectured materials. It is now possible to build micro-lattices, or other complex shapes, using additive manufacturing techniques like Selective Laser Melting (SLM) (Mines et al., 2013), Fused Deposition Modeling (FDM) (Compton and Lewis, 2014) or Electron Beam Melting (EBM) (Suard et al., 2014). For instance, this new flexibility in the manufacturing landscape allows designers to work with more complex material architectures and further explore the material property space (Ashby, 2005). Several authors have adressed the problem of selecting the best architecture for a given problem, either empirically (Sigmund, 2000), or using numerical optimization methods that are coupled with the homogenization analysis of heterogeneous periodic media (Allaire, 2002; Andreassen et al., 2014; Bendsøe and Kikuchi, 1988; Wang et al., 2004). In these previous studies, the goals included: (i) obtaining materials with target or extremal properties that are close to theoretical bounds, or (ii) understanding mechanisms used to achieve a desired behavior or material response. For example, most of the composite or heterogeneous materials design problems related to achieve precribed thermal properties (for instance materials with a low coefficient of thermal expansion) in the literature employ mechanisms similar to those presented in Figure 1. This illustrates which kind of mechanism can control the vertical expansion of a structure consisting in: $a$ ) a standard structure subject to thermal expansion, $b$ ) and $c$ ) a vertical contraction making use of a bending or a bimetallic mechanism, respectively. In this study, we focus on achieving extremal thermoelastic properties (Sigmund and Torquato, 1997) with the level-set topology optimization approach (Allaire et al., 2004; Wang et al., 2003).

As in (Sigmund and Torquato, 1997), most of the studies in multi-material optimization assume a sharp transition of properties across material interfaces. However, there are many cases where this transition is not sharp, but smooth or graded (see Figure 2), for instance Creton et al. (2001) report that the interface in polymers can be strengthened by using anchor chains . Graded interfacial properties are induced by the processing or can be controlled in some cases as in functiontionnally graded materials (Kieback et al., 2003). There are also many cases where this interface transition may be non-monotonic, introducing new material properties that are not provided by the bulk constitutive or phases. For instance, Tan et al. (2016) report non-monotonic variations of hardness accross an interface between two metallic phases and Markworth et al. (1995) report non-monotonic variations of thermal stress across graded interfaces in polymers.

In this study, we investigate the design of hybrid micro-architectured materials with target extremal thermoelastic properties in a shape optimization framework. In particular, we evaluate the effects of graded interfaces between the constitutive materials or phases.The paper is organised as follow: in the first section, we define the shape optimization framework. We describe how an heterogeneous multi-material composite with graded interfaces can be represented (Vermaak et al., 2013), and how the graded interfaces are included in the shape optimization formulation. In the 


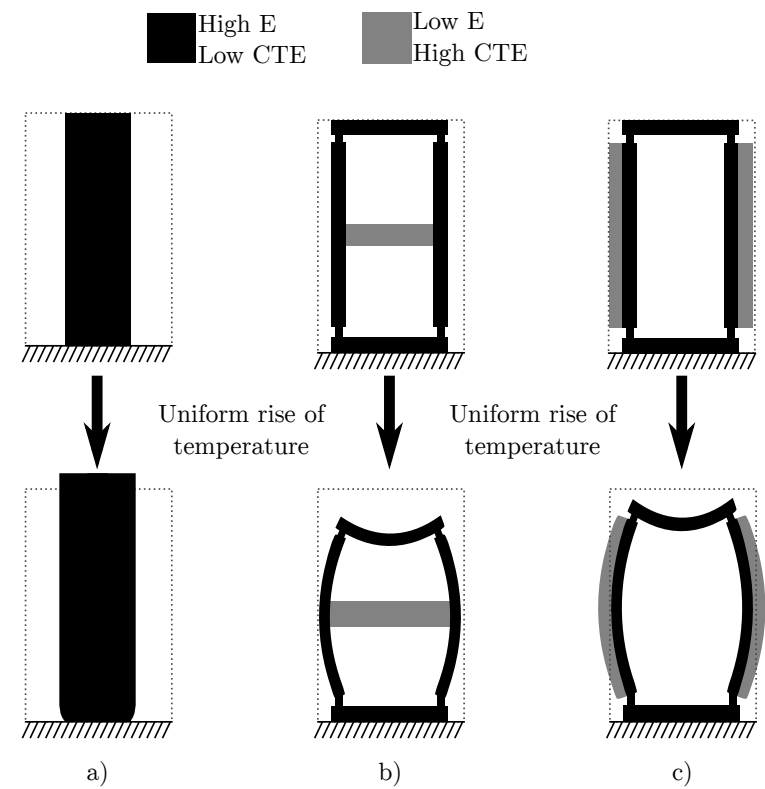

Figure 1: Illustration of conceptual mechanism used to adjust thermal expansion. (i) a monolithic bulk material subject to a uniform temperature rise for reference (ii) first mechanism of thermal expansion control ("three point flexion") and (iii) second mechanism of thermal expansion control ("bi-metallic"). Applications of these mechanisms are presented by Jefferson et al. (2009)

second section, we present the computational aspects of the optimization problem. Results of the optimized microstructure for a selection of extreme thermoelastic target properties are presented in a third section followed by a discussion in section 4. Concluding remarks close the study. 


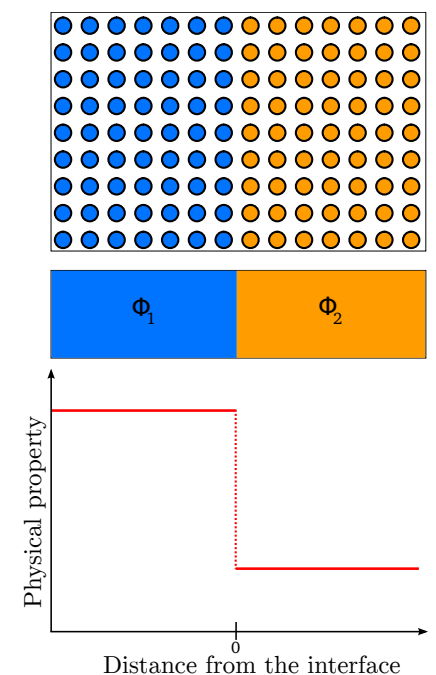

Element of material/phase 2

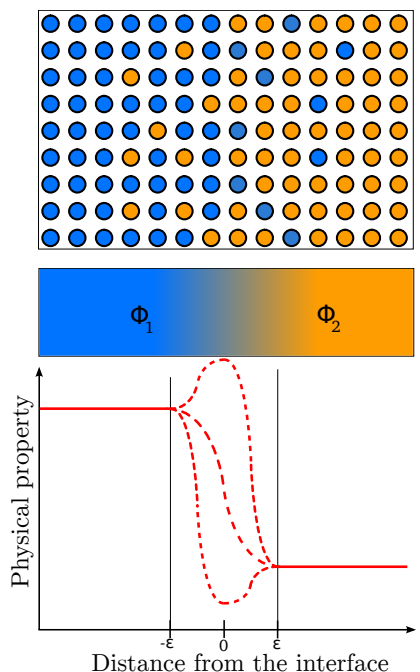

Element of material/phase 1

Figure 2: Scheme of interface transition zone models between materials $\left(\Phi_{1}, \Phi_{2}\right)$. Theoretically, interfaces are infinitely sharp transitions from one material or phase to another, but most of real interfaces exhibit a smooth transition in chemical composition, which can lead to a wide array of properties.

\section{Optimization framework}

This section is an overview of all the key point that should be treated to build a numerical optimization process that takes into account the graded interfaces effects. The basis is a shape optimization code as the one used by Vermaak et al. (2016), adapted to be used with homogenization framework.

\subsection{Calculation of homogenized properties}

We aim at designing periodic multi-material composites with prescribed effective thermoelastic properties that are estimated by periodic homogenization (see Figure 3). We consider a domain made of a periodic unit cell, $Y$, with its periodic lengthscale, $\delta$. We denote $C(x)$ the fourth order elastic tensor and $\alpha(x)$ the second order thermal expansion tensor. The thermal stress tensor is defined as $A(x)=C(x) \alpha(x)$. Note that all of these properties depend on the spatial variable $x$. The linearized thermoelastic system that governed the thermoelastic response of this periodic domain as:

$$
\left\{\begin{array}{cc}
-\operatorname{div}(C(x)(\epsilon(u)-\alpha(x) \Delta T))=0 & x \in \Omega \\
u=0 & x \in \partial \Omega
\end{array},\right.
$$

with $u$ the displacement field and solution of equation (1), $\Delta T$ a uniform temperature field, and $\epsilon(u)$ the strain operator (defined as $\epsilon(u)=\left(\nabla u+\nabla u^{T}\right) / 2$, where ()$^{T}$ denotes the transpose operator).

Using the theory of two-scale asymptotic development (Allaire, 2002), the effective properties 
Periodic domain

$\mathrm{P}(\mathrm{x})$ e.g. $\mathrm{C}(\mathrm{x}), \alpha(\mathrm{x})$ or $\mathrm{A}(\mathrm{x})$

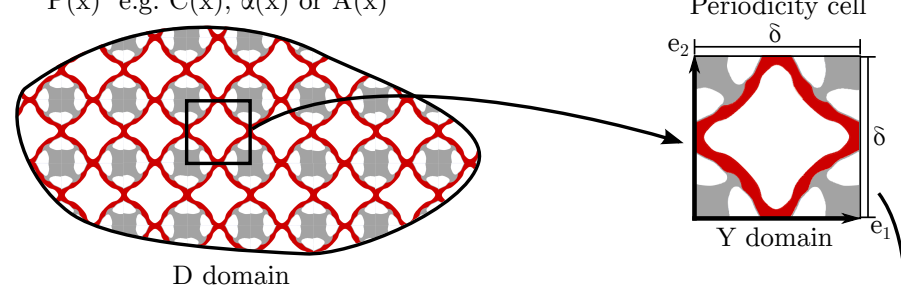

Homogenized continum

$\mathrm{P}^{*}$ e.g. $\mathrm{C}^{*}, \alpha^{*}$ or $\mathrm{A}^{*}$

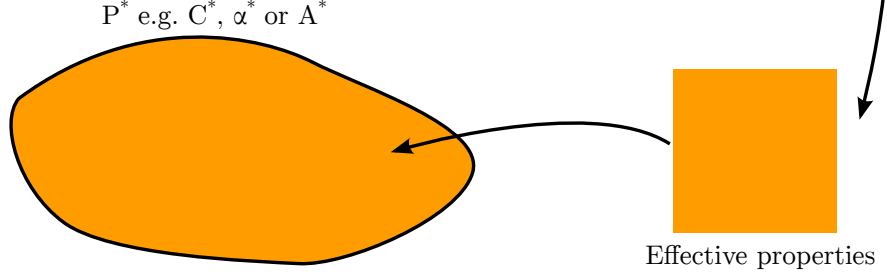

Figure 3: Homogenization scheme for a multi-material or multi-phase periodic composite.

of the periodic heterogeneous medium are derived. The effective elastic tensor, we have

$$
C_{i j k l}^{*}=\int_{Y} C(y)\left(e_{i j}+\epsilon_{y}\left(\omega_{i j}\right)\right) e_{k l} d v,
$$

and for the effective thermal stress tensor

$$
A_{i j}^{*}=\int_{Y} C(y)\left(e_{i j}-\epsilon\left(\omega_{i j}\right)\right)\left(\alpha(y)-\epsilon_{y}\left(\omega_{\theta}\right)\right) d v
$$

where $y=\frac{x}{\epsilon}$ (the so-called "fast" variable that denotes the scale separation between the heterogeneous domain and the unit cell $Y$ ), and where $\omega_{i j}$ and $\omega_{\theta}$ are the solutions of the so-called "cell problems". The cell problems describe the response of the heterogeneous periodic unit cell to unit strain

$$
e_{i j}=\frac{e_{i} \otimes e_{j}+e_{j} \otimes e_{i}}{2},
$$

where $e_{i}$ and $e_{j}$ are orthogonal vectors of the spatial basis (in two dimensions, $1 \leq i \leq 2$ ). Thus, each $\omega_{i j}$ is the solution of the cell problem when $e_{i j}$ is the applied unit deformation, in a similar way, $\omega_{\theta}$ is the the solution of the cell problem where the unit deformation is $\alpha$.

The fields $\omega_{i j}$ are the solution for the cell problems

$$
\left\{\begin{array}{cl}
-\operatorname{div}_{y}\left(C(y)\left(e_{i j}+\epsilon_{y}\left(\omega_{i j}(y)\right)\right)=0\right. & \text { in } Y \\
\omega_{i j}(y) & Y-\text { periodic }
\end{array},\right.
$$

and in the same way, $\omega_{\theta}$ corresponds to the thermal cell problem defined as

$$
\left\{\begin{array}{cl}
-\operatorname{div}_{y}\left(C(y)\left(\alpha(x)-\epsilon_{y}\left(\omega_{\theta}(y)\right)\right)=0\right. & \text { in } Y \\
\omega_{\theta}(y) & Y \text {-periodic }
\end{array} .\right.
$$


With an expression for the effective elasticity tensor, $C^{*}$, and the effective thermal stress tensor, $A^{*}$, we can also derive the effective thermal expansion tensor

$$
\alpha^{*}=\left(C^{*}\right)^{-1}: A^{*} .
$$

The foregoing formulation defines the homogenization procedure.

\subsection{Formulation of the optimization problem}

In this study, we propose an optimization methodology to design isotropic composites with extremal thermal expansion coefficients (CTE) and a minimum stiffness that is prescribed through a minimum bulk modulus. Composites with extremal CTE are of interest for a number of industrial applications and their range of use is extended by requiring baseline stiffness properties. Without a minimum stiffness constrain, optimized mechanisms will contain a lot of hinges, leading to appropriate mechanisms but poor mechanical properties (thin parts that are expected to be weaken the structure). Although some authors have already addressed this problem (Sigmund and Torquato, 1997; Wang et al., 2004), we extend the previous frameworks in order to account for interface effects between the constitutive material phases. We evaluate how interface effects offers new possibilities for exploring the material's property space and especially the design of architectured materials (Vermaak et al., 2016). We focus on the following two optimization problems which are restricted to $2 \mathrm{D}$ cases for sake of simplicity.

i ) Find the lowest possible effective thermal expansion coefficient for a composite,

$$
\min _{\Omega} \mathcal{J}(\Omega)=\alpha_{11}^{*}+\alpha_{22}^{*}=\operatorname{tr}\left(\alpha^{*}\right)
$$

ii ) Achieving the highest possible effective thermal expansion coefficient for a composite,

$$
\min _{\Omega} \mathcal{J}(\Omega)=-\alpha_{11}^{*}-\alpha_{22}^{*}=-\operatorname{tr}\left(\alpha^{*}\right)
$$

The lowest or highest possible values are constrained by the theoretical thermoelastic bounds (described below)(Gibiansky and Torquato, 1997) which provide extreme frontiers for isotropic effective CTE $\alpha^{*}$ and the bulk modulus $K^{*}$. However during the optimization iterations, the design does not warranty isotropic effective properties. Thus, we could estimate a bulk modulus by considering the spherical part of the effective elastic moduli tensor. We adopt a simpler and practical approach consisting in deriving $K$ from the volumetric strain induced by a hydrostatic loading as

$$
K=\frac{\operatorname{tr}(\overline{\bar{\sigma}})}{\operatorname{tr}(\overline{\bar{\epsilon}})}, \text { with } \overline{\bar{\sigma}}=-\mathrm{P} \overline{\bar{I}}
$$

where $\overline{\bar{I}}$ is the second order identity tensor and $\operatorname{tr}()$ denotes the trace. If the composite is isotropic, we have $K=\kappa$. In order to obtain isotropic microstructures for a proper comparison with the theroetical bounds, we define an isotropy error estimator. Let $C^{i s o}$ be the isotropized elastic tensor of a composite defined as

$$
\left\{\begin{array}{l}
C_{1111}^{i s o}=C_{2222}^{i s o}=\frac{C_{1111}^{*}+C_{2222}^{*}}{2} \\
C_{1122}^{i s o}=C_{1122}^{*} \\
C_{1112}^{i s o}=0 \\
C_{2212}^{i s o}=0 \\
C_{1212}^{i s o}=C_{1111}^{i s o}-\frac{C_{1122}^{*}}{2}
\end{array} .\right.
$$


In this way, $C^{i s o}$ is an isotropic equivalent of the effective elastic tensor, $C^{*}$. To force rhe isotropic symmetry, we minimize the difference between $C^{*}$ and $C^{i s o}$ in order to design an isotropic composite. An isotropy error estimator is defined as

$$
e r r_{i s o}^{2}=\frac{\sum_{i j k l}\left(C_{i j k l}^{*}-C_{i j k l}^{i s o}\right)^{2}}{\left(C_{1111}^{i s o}\right)^{2}}
$$

This estimator could also be added as a parameter to be constrained in the optimization problem, as it is done by Andreassen et al. (2014).

In a same manner, the isotropic symmetry of thermal expansion tensor $\alpha^{*}$ at the end of the optimization process is not ensured. It is possible to define another error estimator for the CTE isotropy. However, we prefer to force the isotropy of $\alpha^{*}$ by definning a symmetry on geometry. The use of a symmetry on the unit cell reduces the constraints of the optimization problem.

Thus, the optimization problem corresponding to equation (8) reads

$$
\begin{array}{ll}
\min _{\Omega} & \alpha_{11}^{*}+\alpha_{22}^{*} \\
\text { s.t. } & K>K_{\min } \\
& \text { err }_{\text {iso }}<\text { err }_{\max } \\
& V_{i}=V_{i, \text { prescribed }} \\
& 4^{\text {th } \text { order rotation symmetry }}
\end{array}
$$

The above description defines the objective and constraints of the problem under consideration. Now that we have defined the problem and the homogenization framework, we present the interpolation scheme for the physical interfacial properties: the elastic tensor, $C(y)$, and thermal expansion tensor, $\alpha(y)$.

\subsection{Level-set method}

The level-set method is adopted to describe the spatial distribution of materials because of its efficiency in tracking evolving domains (Osher and Sethian, 1988). The level-set method's ability to handle topological changes in a natural way also makes it a robust approach for performing topology optimization by keeping a geometric description of the evolving shape. Since the first publications on the topic (Allaire et al., 2004; Sethian and Wiegmann, 2000; Wang et al., 2003), the level-set method for topology optimization continues to gain popularity, providing solutions to problems that cannot be adressed by conventional density approaches, in particular for the account for interface effects under consideration here.

In the level-set framework, the boundary of a domain, $\Omega$, is defined via the zero level-set of an auxiliary function, $\phi$ (see Figure 4 ) as

$$
\left\{\begin{aligned}
\phi(x)=0 & \leftrightarrow x \in \partial \Omega \cap D, \\
\phi(x)<0 & \leftrightarrow x \in \Omega, \\
\phi(x)>0 & \leftrightarrow x \in(D \backslash \Omega) .
\end{aligned}\right.
$$

In order to represent an evolving domain, $\Omega$, we update the level-set function $\phi(x)$ by solving a Hamilton-Jacobi transport equation using an explicit "upwind" finite difference scheme (Sethian, 1999). The level-set function is advected with a normal velocity field that describes the velocity of the interface, $\partial \Omega$, along its normal direction. The advection time step is chosen such that it verifies the Courant-Friedrichs-Lewys (CFL) stability condition. 


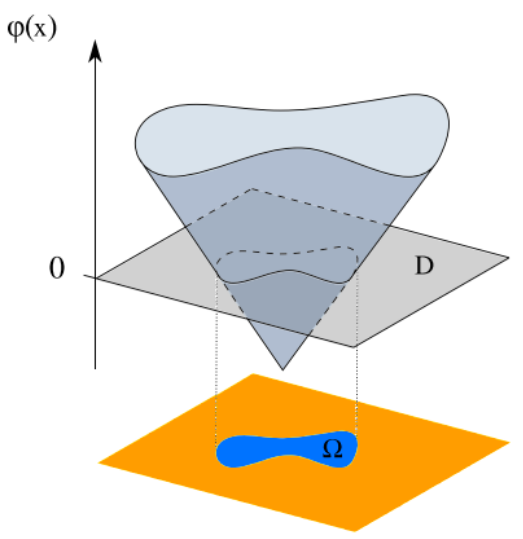

Figure 4: Level-set description of a domain, $\Omega \subset D$.

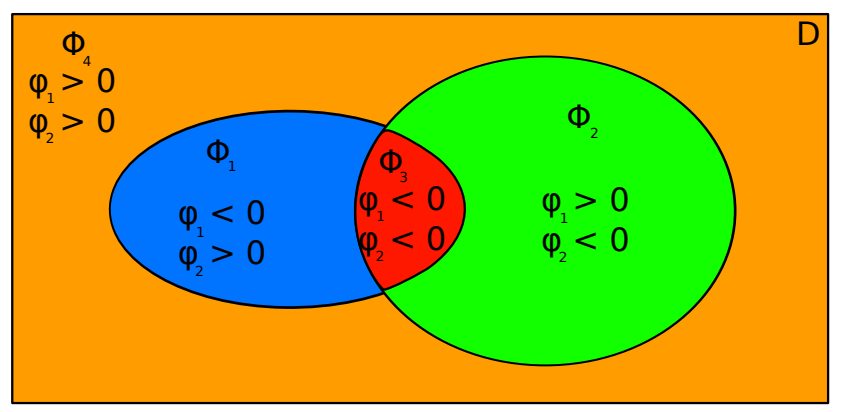

Figure 5: Definition of four materials domains that are represented by the regions $\Phi_{1,2,3,4}$ using two level-set functions, $\phi_{1}$ and $\phi_{2}$.

Using one level-set function allows the description of two domains in space, i.e. $\Omega$ and $D \backslash \Omega$. In our study, we account for more than two materials or phases in space. It is then necessary to handle more than one level-set function. As presented in Allaire et al. (2014); Vermaak et al. (2016), it is possible to represent $2^{N}$ material domains, $\Phi_{i}$, using $N$ level-set functions, $\phi_{j}$, (see Figure 5). The different materials are defined by combining the values of the level-set functions as

$$
\left\{\begin{array}{ll}
\phi_{1}(x)<0 \text { and } \phi_{2}(\mathrm{x})>0 & , x \in \Phi_{1} \\
\phi_{1}(x)>0 \text { and } \phi_{2}(\mathrm{x})<0 & , x \in \Phi_{2} \\
\phi_{1}(x)<0 \text { and } \phi_{2}(\mathrm{x})<0 & , x \in \Phi_{3} \\
\phi_{1}(x)>0 \text { and } \phi_{2}(\mathrm{x})>0 & , x \in \Phi_{4}
\end{array} .\right.
$$

\subsection{Definition of the interpolation scheme for the thermoelastic proper- ties}

In this section, we propose an interpolation scheme that accounts for graded interfaces between different phases in an heterogeneous media. Using the multi-material representation (Section 2.3), we can construct the signed distance functions, $d_{\Omega_{1}}$ and $d_{\Omega_{2}}$, of the domains, $\Omega_{1}$ and $\Omega_{2}$, that are described by the level-set functions, $\phi_{1}$ and $\phi_{2}$, respectively. We first focus on the definition of the interface between materials $\Phi_{1}$ and $\Phi_{4}$. According to Figure 5, this corresponds to a change of sign for $\phi_{1}$. We use an interpolation function, $h_{i}$ as in Allaire et al. (2014)

$$
h_{i}=\left\{\begin{array}{ll}
0 & , d_{\Omega_{i}}<-\epsilon \\
\frac{1}{2}+\frac{d_{\Omega_{i}}}{2 \epsilon}+\frac{1}{2 \pi} \sin \frac{\pi d_{\Omega_{i}}}{\epsilon} & ,\left|d_{\Omega_{i}}\right|<\epsilon \\
1 & , d_{\Omega_{i}}>\epsilon
\end{array},\right.
$$

where $\epsilon$ is the half thickness of the interface zone. We adopt this expression because it is smooth and differentiable.

Assuming that $\phi_{2}>0$, the Young's modulus, $E$, at a point $x$ in space is

$$
E(x)=E_{4} h_{1}+E_{1}\left(1-h_{1}\right),
$$


where $E_{i}$ the Young's modulus of the $\mathrm{i}^{\text {th }}$ material/phase. Figure 6 presents this interpolation scheme along a transition between materials $\Phi_{1}$ and $\Phi_{4}$. The same interpolation can be calculated for each
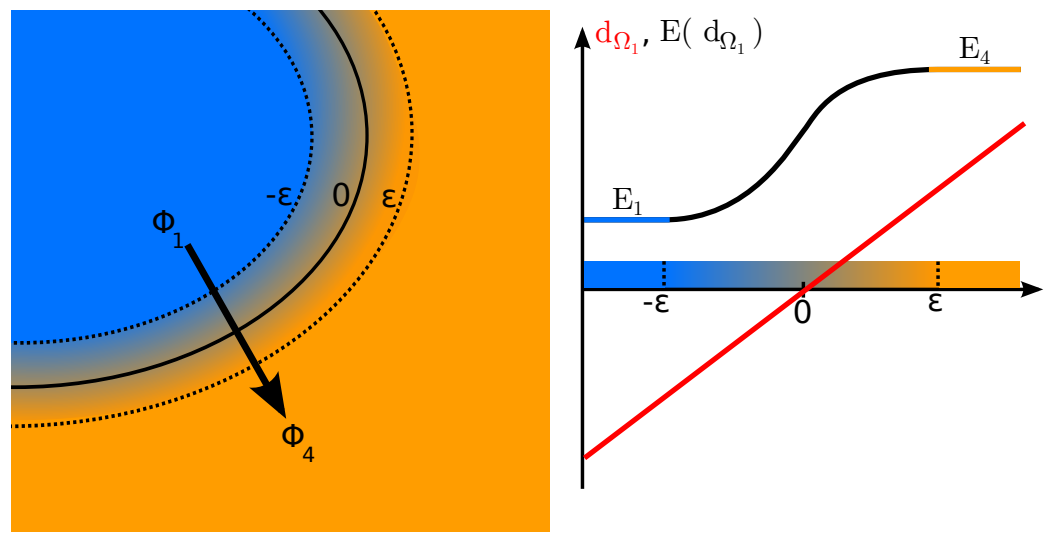

Figure 6: Model for graded interfaces. The material property related to stiffness, Young's modulus or $E$, is defined as a function of the signed distance, $d_{\Omega} . E_{1}$ and $E_{4}$ define Young's Modulus for phase $\Phi_{1}$ and $\Phi_{4}$ respectively. We see that for $d_{\Omega_{1}}<-\epsilon$ we have $E\left(d_{\Omega_{1}}\right)=E_{1}$, for $d_{\Omega_{1}}>\epsilon$ we have $E\left(d_{\Omega_{1}}\right)=E_{4}$, and for $-\epsilon \leq d_{\Omega_{1}} \leq \epsilon, E\left(d_{\Omega_{1}}\right)$ is inteprolated using the equations (17) and (16).

material interface transition. In more general terms, one can define a global interpolation scheme that handles all of the transitions between materials or phases simultaneously:

$$
\begin{aligned}
P(x)= & P_{1}\left(1-h_{1}\right) h_{2}+P_{2} h_{1}\left(1-h_{2}\right) \\
& +P_{3}\left(1-h_{1}\right)\left(1-h_{2}\right)+P_{4} h_{1} h_{2},
\end{aligned}
$$

where $P$ represents a mechanical or a thermal property. The subscript in $P$ denotes the index of the material under consideration. Figure 7 shows an example of the interpolation scheme defined by equation (18).

This interpolation scheme (18) is similar to the one used by Vermaak et al. (2013). In the present work, instead of varying the interpolation function for the properties as was done in Vermaak et al. (2013), we introduce a new region that represents the interface, denoted by the subscript "int". We define $P_{\text {int }}$ the property of the interface between materials $\Phi_{1}$ and $\Phi_{4}$, and we use the following interpolation scheme (see Figure 8):

$$
\begin{aligned}
P= & P_{1}\left(1-h^{m}\right) h_{2}+P_{2} h_{1}\left(1-h_{2}\right)+ \\
& P_{3}\left(1-h_{1}\right)\left(1-h_{2}\right)+P_{4} h^{p} h_{2} \\
& P_{\text {int }}\left(h^{m}\right) h_{2}+P_{\text {int }}\left(1-h^{p}\right) h_{2},
\end{aligned}
$$

where $h_{m}$ and $h_{p}$ are two functions:

$$
h^{m}= \begin{cases}0 & , d_{\Omega_{1}}<-\epsilon \\ 1+\frac{d_{\Omega_{1}}}{\epsilon}+\frac{1}{2 \pi} \sin \frac{2 \pi\left(d_{\Omega_{1}}+\frac{\epsilon}{2}\right)}{\epsilon} & ,-\epsilon \leq d_{\Omega_{1}}<0,\end{cases}
$$

and

$$
h^{p}=\left\{\begin{array}{ll}
\frac{d_{\Omega_{1}}}{\epsilon}+\frac{1}{2 \pi} \sin \frac{2 \pi\left(d_{\Omega_{1}}-\frac{\epsilon}{2}\right)}{\epsilon} & , 0<d_{\Omega_{1}} \leq \epsilon . \\
1 & , d_{\Omega_{1}}>\epsilon
\end{array} .\right.
$$




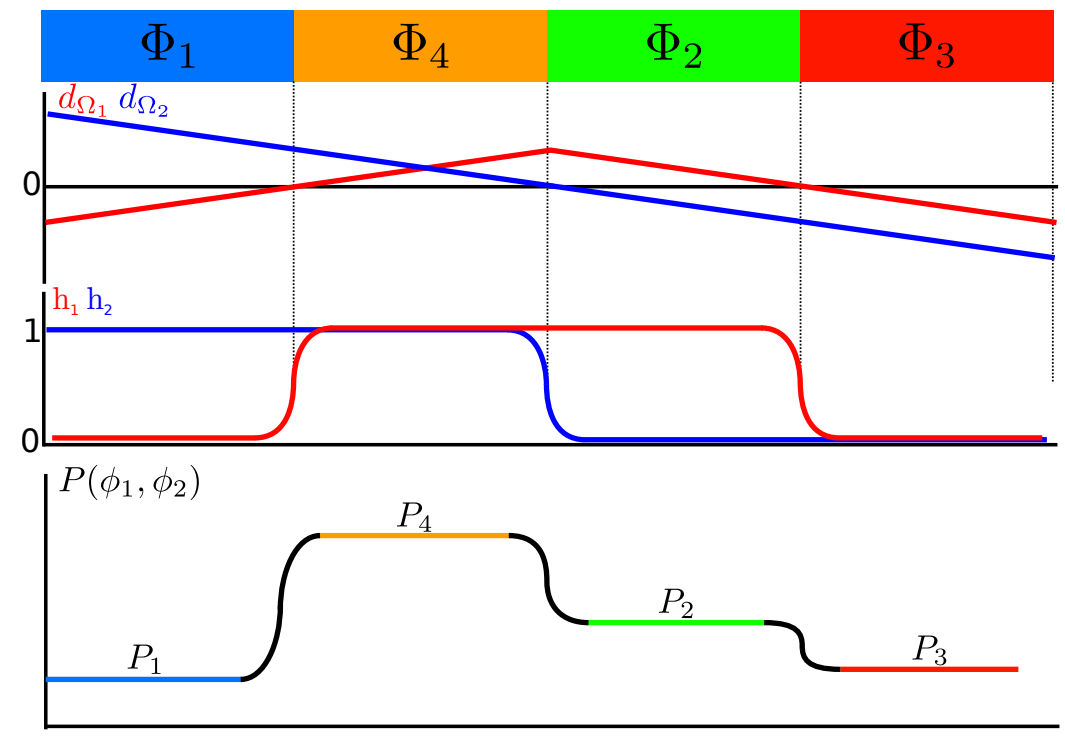

Figure 7: Example of the interpolation scheme for a 1D multi-material in the case of a monotonic graded interface between two adjacent bulks. $P_{i}$ corresponds to a property of phase $\Phi_{i}$, (elastic moduli, thermal expansion). We see how the scheme defined in the equation (18) interpolates a given property $P$ depending on the signed distance functions $d_{\Omega_{1}}$ and $d_{\Omega_{2}}$. The functions $h_{1}$ and $h_{2}$ used in the equation (18) are also presented.

The same interpolation scheme (19) could be used to handle non-monotonic interfaces between any bulks. In order to remain consistent with the interpolation function, $h_{i}$, defined in equation (16), we choose to use the same expression of interpolation function for the description of the nonmonotonic interfaces. Note that because of the interpolation choice, the dependency on the spatial variable is replaced by one on the signed distance functions. Thus, we change the notation $C(y)$ and $\alpha(y)$ to $C\left(d_{\Omega_{1}}, d_{\Omega_{2}}\right)$ and $\alpha\left(d_{\Omega_{1}}, d_{\Omega_{2}}\right)$, respectively.

Having the spatial distribution of the interpolated thermoelastic properties, the effective properties can be derived by homogenization. In order to complete the optimization protocol, it is now necessary to compute the shape derivatives of these effective properties.

\subsection{Shape derivative}

We first compute the shape derivatives $C_{i j k l}^{\prime}(\theta)$ and $A_{i j}^{\prime}(\theta)$, that are used in the expression of the functional we want to optimize $\left(K^{*}, \alpha^{*}, \operatorname{err}_{\text {iso }}\right)$ defined in Section 2.2. We adopt the interpolation scheme presented in section 2.4 , with the effective elastic moduli are derived from

$$
C_{i j k l}^{*}=\int_{Y} C\left(d_{\Omega_{1}}, d_{\Omega_{2}}\right)\left(e_{i j}+\epsilon_{y}\left(\omega_{i j}\right)\right) e_{k l} d y,
$$

where $\omega_{i j}$ is the solution of the cell problem (equation 5). Note that $\omega_{i j}$ depends on both $\Omega_{1}$ and $\Omega_{2}$. Its direct derivation is not straightforward. Therefore, we adopt Céa's shape derivation method (Céa, 1986) for its estimation. 


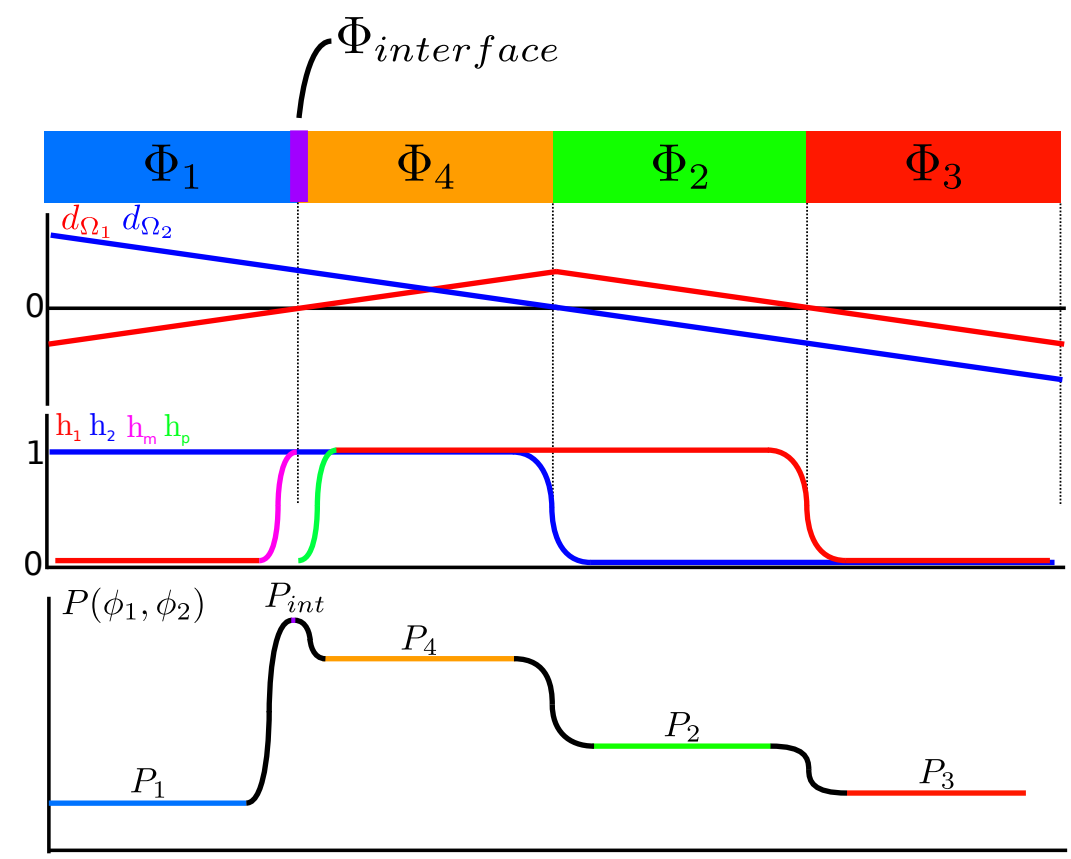

Figure 8: Example of the interpolation scheme for a $1 \mathrm{D}$ multi-material in the case of a nonmonotonic interface. $P_{i}$ indicates a property of phase $\Phi_{i}$, (elastic moduli, thermal expansion). We see how the scheme defined in the equation (21) interpolates a given property $P$ depending on the signed distance functions $d_{\Omega_{1}}$ and $d_{\Omega_{2}}$. The additional functions $h^{m}$ and $h^{p}$ used in the equation (21) are also represented.

In particular, to caluclate the shape derivative of $C_{i j k l}^{*}$, we formuate the Lagrangian of the problem

$$
\begin{aligned}
& \mathcal{L}\left(\Omega_{1}, \Omega_{2}, \hat{\omega}_{i j}, \hat{\eta}_{i j}\right)= \\
& \int_{Y} C\left(d_{\Omega_{1}}, d_{\Omega_{2}}\right)\left(e_{i j}+\epsilon_{y}\left(\hat{\omega}_{i j}\right)\right) e_{k l} d y+ \\
& \int_{Y} C\left(d_{\Omega_{1}}, d_{\Omega_{2}}\right)\left(e_{i j}+\epsilon_{y}\left(\hat{\omega}_{i j}\right)\right): \epsilon_{y}\left(\hat{\eta}_{i j}\right) d y
\end{aligned}
$$

where $\hat{\omega}_{i j}$ and $\hat{\eta}_{i j}$ are vector fields that are independent of $\Omega_{1}$ and $\Omega_{2}$. By differentiating the Lagrangian with respect to $\hat{\eta}_{i j}$, along the direction $\phi$, and by assuming that this derivative is zero at the optimal point $\left(\Omega_{1}, \Omega_{2}, \omega_{i j}^{*}, \eta_{i j}^{*}\right)$, we obtain

$$
\begin{aligned}
& \frac{\partial \mathcal{L}\left(\Omega_{1}, \Omega_{2}, \omega_{i j}^{*}, \eta_{i j}^{*}\right)}{\partial \hat{\eta}_{i j}}(\phi)= \\
& \int_{Y} C\left(d_{\Omega_{1}}, d_{\Omega_{2}}\right)\left(e_{i j}+\epsilon_{y}\left(\omega_{i j}^{*}\right)\right): \epsilon_{y}(\phi) d y \\
& =0 .
\end{aligned}
$$

from which we derive that $\omega_{i j}^{*}$ is the solution of the cell problem (equation 5). 
Taking the derivative of the Lagrangian with respect to $\hat{\omega}_{i j}$, along the direction $\phi$, and at the optimal point where the derivative is equal to zero, we obtain

$$
\begin{aligned}
& \frac{\partial \mathcal{L}\left(\Omega_{1}, \Omega_{2}, \omega_{i j}^{*}, \eta_{i j}^{*}\right)}{\partial \hat{\omega}_{i j}}(\phi)= \\
& \int_{Y} C\left(d_{\Omega_{1}}, d_{\Omega_{2}}\right)\left(e_{k l}+\epsilon_{y}\left(\omega_{k l}^{*}\right)\right): \epsilon_{y}(\phi) d y+ \\
& \int_{Y} C\left(d_{\Omega_{1}}, d_{\Omega_{2}}\right)\left(\epsilon_{y}\left(\eta_{i j}^{*}\right)\right): \epsilon_{y}(\phi) d y=0 .
\end{aligned}
$$

The first term in equation (25) corresponds to the weak formulation of the cell problem (equation 5 ), then it vanishes at the optimal point. The second term leads to $\eta_{i j}^{*}=0$. Thus the problem is self-adjoint.

The shape derivatives of the effective elastic moduli are equal to those of the Lagrangian function at the optimal point $\left(\Omega_{1}, \Omega_{2}, \omega_{i j}^{*}, \eta_{i j}^{*}\right)$ (Allaire, 2007), for any variation of the domains, $\Omega_{1}$ and $\Omega_{2}$, along a direction $\theta_{1}$ and $\theta_{2}$, respectively

$$
\begin{aligned}
& C_{i j k l}^{*^{\prime}}\left(\Omega_{1}, \Omega_{2}\right)\left(\theta_{1}\right)=\mathcal{L}^{\prime}\left(\Omega_{1}, \Omega_{2}, \omega_{i j}^{*}, \eta_{i j}^{*}\right)\left(\theta_{1}\right) \\
& C_{i j k l}^{*^{\prime}}\left(\Omega_{1}, \Omega_{2}\right)\left(\theta_{2}\right)=\mathcal{L}^{\prime}\left(\Omega_{1}, \Omega_{2}, \omega_{i j}^{*}, \eta_{i j}^{*}\right)\left(\theta_{2}\right)
\end{aligned} .
$$

In the sequel, we illustrate how the shape derivative proceeds for the domain $\Omega_{1}$, the same holds

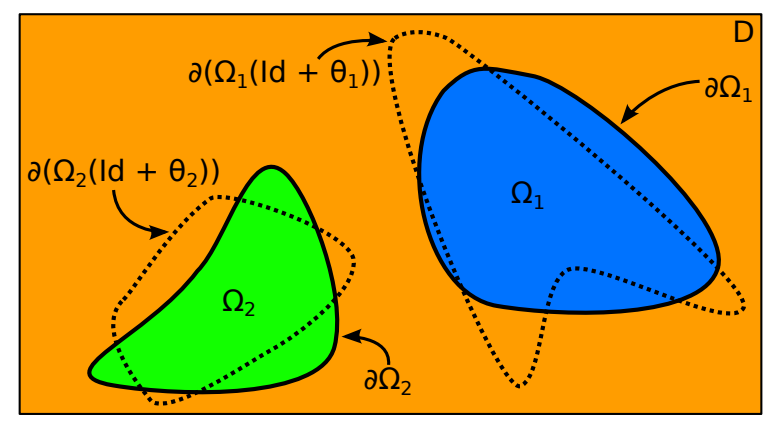

Figure 9: Perturbation of the boundary, $\partial \Omega$, by a small vector field, $\bar{\theta}$, that defines the new domain, $\Omega(I d+\bar{\theta})$.

for $\Omega_{2}$. From the expression od the Lagrangian in (23) and the relation (26), the shape derivative is

$$
\begin{aligned}
& \int_{i j k l}^{*^{\prime}}\left(\Omega_{1}, \Omega_{2}\right)\left(\theta_{1}\right)= \\
& \int_{Y}^{\prime}\left(d_{\Omega_{1}}^{\prime}\left(\theta_{1}\right) \frac{\partial C\left(d_{\Omega_{1}}, d_{\Omega_{2}}\right)}{\partial d_{\Omega_{1}}} \cdot\left(\epsilon\left(\omega_{i j}\right)+e_{i j}\right) e_{k l}\right) d v,
\end{aligned}
$$

where $d_{\Omega_{1}}^{\prime}\left(\theta_{1}\right)$ is the shape derivative of the signed distance function, $d_{\Omega_{1}}$.

Similarly, one can obtain the shape derivative for the thermal stress

$$
\begin{aligned}
& A_{i j}^{*^{\prime}}\left(\Omega_{1}, \Omega_{2}\right)\left(\theta_{1}\right)= \\
& \int_{Y}\left(d_{\Omega_{1}}^{\prime}\left(\theta_{1}\right) \frac{\partial C}{\partial d_{\Omega_{1}}} \cdot\left(\alpha(y)-\epsilon\left(\omega_{i j}^{\theta}\right)\right) e_{i j}\right) d v .
\end{aligned}
$$




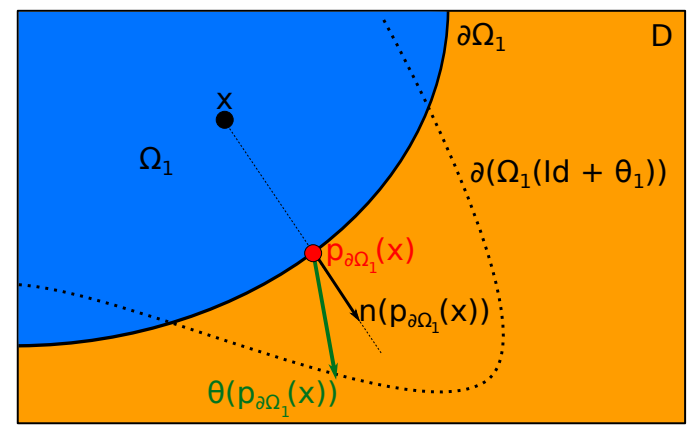

Figure 10: Illustration of the terms used in the expression of the shape derivative of the signed distance function.

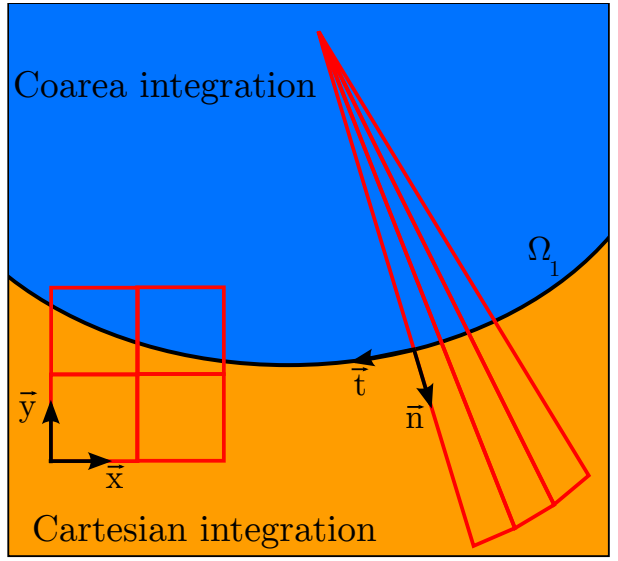

Figure 11: Scheme for the integration performed along rays and a comparison to Cartesian integration.

In (27) and (28), the shape derivative of the signed distance function $d_{\Omega_{1}}^{\prime}\left(\theta_{1}\right)$ is taken as in Allaire et al. (2014). Some additional steps are still required to compute the shape derivative explicitly. For instance, obtaining explicit expressions for the shape derivatives of the signed distance functions, $d_{\Omega_{1}}$ and $d_{\Omega_{2}}$. This step is addressed in Allaire et al. (2014), and the expression is

$$
d_{\Omega_{1}}^{\prime}\left(\theta_{1}\right)(x)=-\theta\left(p_{\partial \Omega_{1}}(x)\right) \cdot n\left(p_{\partial \Omega_{1}}(x)\right),
$$

in which, for any point $x \in Y, p_{\partial \Omega}(x)$ denotes its orthogonal projection on the boundary $\partial \Omega_{1}$, and $n\left(p_{\partial \Omega_{1}}(x)\right)$ denotes the exterior normal at this projected point (illustrated in Figure 10).

In order to obtain a descent direction defined along the boundary of the shape, we apply the coarea formula (Allaire et al., 2014). This essentially consists in splitting the integration over the domain $Y$ into two separate integrations: one along the boundary and another along the rays emerging from the boundary (see (Allaire et al., 2014)). Figure 11 illustrates this coarea integration schematically.

After simplifaction of the coarea formula (see (Allaire et al., 2014)), the Jacobian-free expression of the shape derivative of the effective elastic moduli tensor becomes

$$
\begin{aligned}
& C_{i j k l}^{*^{\prime}}\left(\theta_{1}\right)= \\
& \int_{\partial \Omega_{1}} d_{\Omega_{1}}^{\prime}\left(\theta_{1}\right) \int_{\text {ray }}\left(\frac{\partial C}{\partial d_{\Omega_{1}}} \cdot\left(\epsilon\left(\omega_{i j}\right)+e_{i j}\right) e_{k l}\right) d v .
\end{aligned}
$$

Similarly,that for the effective thermal stress tensor is

$$
\begin{aligned}
& A_{i j}^{*^{\prime}}\left(\theta_{1}\right)= \\
& \int_{\partial \Omega_{1}} d_{\Omega_{1}}^{\prime}\left(\theta_{1}\right) \int_{\text {ray }}\left(\frac{\partial C}{\partial d_{\Omega_{1}}} \cdot\left(\alpha(y)-\epsilon\left(\omega_{i j}^{\theta}\right)\right) e_{i j}\right) d v .
\end{aligned}
$$

At this point, all the required shape derivatives can be computed. We now present the optimization algorithm used to solve the optimization problem. 


\subsection{Optimization algorithm}

Now all of the bricks for the optimization problem are defined. We assemble these to set the optimization protocol. The algorithm is as follow (see Figure 12):

1. Initialize the level-set function.

2. Evaluate the objective function and constraints, and calculate their shape derivatives.

3. Select a descent direction (using shape gradients of the objective function and any constraints). Regularize the velocity field by solving a variational problem (Allaire et al., 2014, 2004). This step is done using a linear programming algorithm.

4. Check the convergence criteria. This includes checking the norm of the velocity field $V^{k}$ and stopping the procedure if it becomes smaller than a given value $V_{\min }$.

5. Advect the level-set functions using a Hamilton-Jacobi transport equation, then return to Step 2.

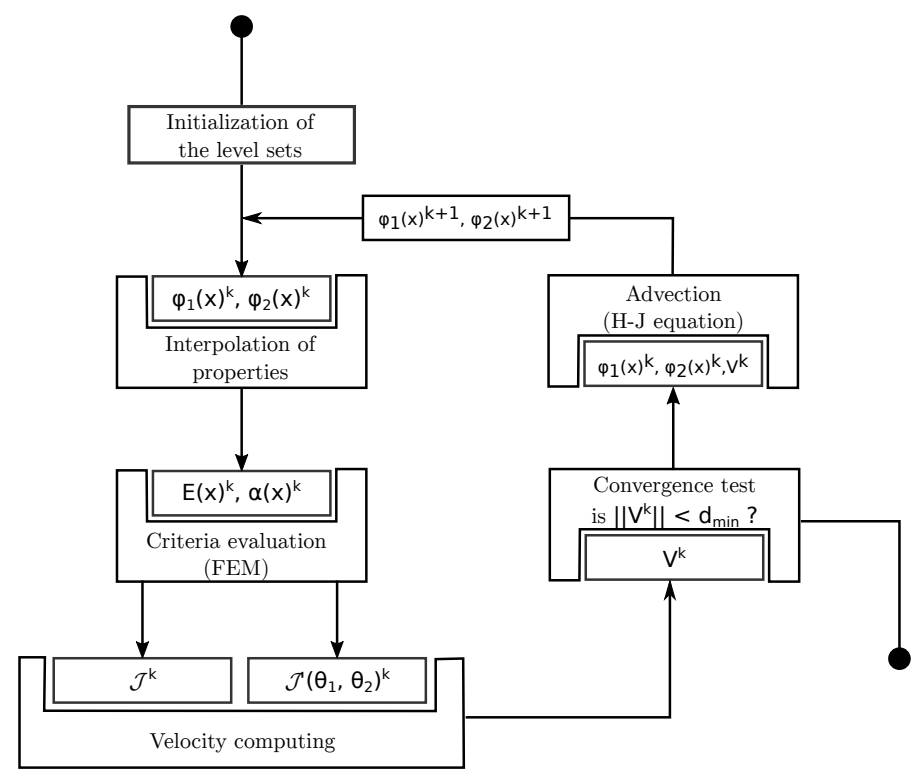

Figure 12: Description of the optimization algorithm used in this study. $V^{k}$ is the velocity field used to advect the level sets (Sethian, 1999).

\section{Results}

The optimization problem is defined by the set of equation (13) and aims at finding the heterogeneous microstructure that meets prescribed extremal properties. Gibiansky and Torquato (1997) have previously established theoretical bounds for the thermoelastic properties of isotropic composites. An example of such bounds is shown in Figure 13 using the same material properties and 


\begin{tabular}{|c|c|c|c|}
\hline Property & phase 1 & phase $2 \& 3$ & phase 4 \\
\hline \hline$E[\mathrm{GPa}]$ & 0.91 & $0.91 \times 10^{-4}$ & 0.91 \\
\hline$\nu$ & 0.3 & 0.3 & 0.3 \\
\hline$\alpha[\mathrm{ppm} / \mathrm{K}]$ & 1 & 1 & 10 \\
\hline Vol. fraction & 0.25 & 0.5 & 0.25 \\
\hline Color & black & white & gray \\
\hline
\end{tabular}

Table 1: Material properties and parameters used for calculations (Young's modulus, E, Poisson's ratio, $\nu, \mathrm{CTE}, \alpha$, and prescribed volume fraction).

volume fractions as in Sigmund and Torquato (1997) (Table 1). The constitutive phases are made of two region with identical elastic moduli but different CTE. These are denoted as 1 and 4 in Table 1 (note that having two bulks and one erzatz of void imply the use of 2 level sets functions). Their volume fraction is 0.25 , the rest of the volume is void that is refered to phase 2 and 3 in Table 1.

We now attempt to design architectured composites of which thermoelastic properties are closed to these theoretical bounds with our optimization protocol.

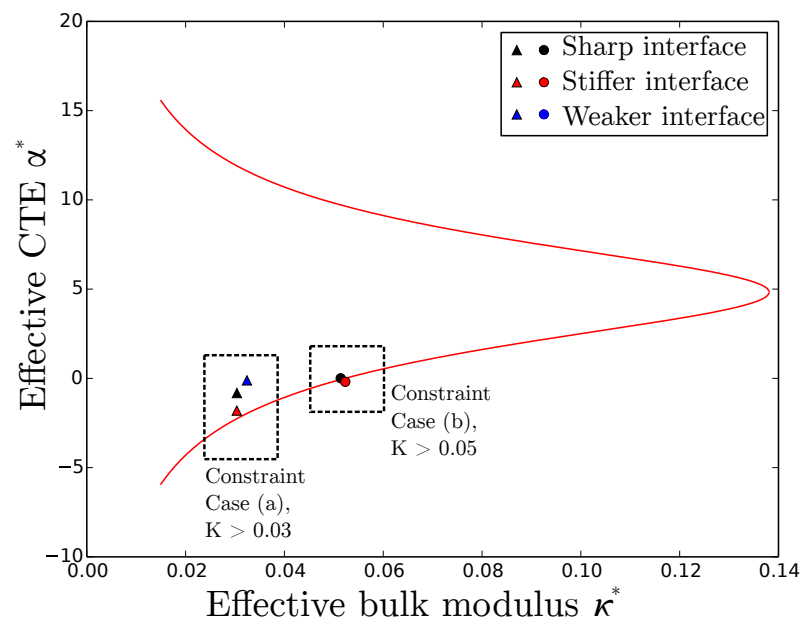

Figure 13: Theoretical bounds on the effective coefficient of thermal expansion (CTE) as a function of the effective bulk modulus (as presented in Gibiansky and Torquato (1997)). All computations were made with the material properties given in Table 1. Each of the results plotted in this chart represent the best design selected from 96 simulations that were initialized slightly differently (see Section 4.1). 
The optimization problem defined in (13) is practically addressed as follow

\begin{tabular}{clll}
\hline search & & \multicolumn{1}{c}{ isotropizing } \\
$\min _{\Omega}$ & $\alpha_{11}^{*}+\alpha_{22}^{*}$ & & $\min _{\Omega} \quad \operatorname{err}_{\text {iso }}^{2}$ \\
& $K^{*}>K_{\text {min }}$ & $\rightarrow$ & $\alpha_{i i}^{*}<\alpha_{\text {ref }}$ \\
$V_{1}=0.25$ & & $K^{*}>K_{\text {min }}$ \\
$V_{4}=0.25$ & & $V_{1}=0.25$ \\
& & $V_{4}=0.25$
\end{tabular}

We first look for an optimization step "search" intended to provide a first solution without taking into account the isotropy constrain. The CTE estimated at this step is denoted $\alpha_{\text {ref }}$. Then, the step "isotropizing" aims at defining the isotropic effective properties without "degrading too much" the results obtained after the "search" step.

We next define two case studies which differ only in the minimum value prescribed for the bulk modulus, $K_{\min }$.

- Case $(a)$, the minimum bulk modulus is set to $K_{\min }=0.03$, refered as the "compliant" case.

- Case $(b)$, the minimum bulk modulus is set to $K_{\min }=0.05$, refered as the "stiff" case.

In the following sections, we report and comment the results obtained for several different interface profiles between phases 1 and 4 for the two case studies $(a)$ and $(b)$. The interface properties are derived from the interpolation defined in (18) and (19), for monotonic and non-monotonic profiles respectively. The thickness profile is calculated over four elements, the unit cell being discretized by a $120 \times 120$ quadrangle elements mesh.

\subsection{Monotonic interface profile}

In this section, we present the closest optimized design to the theoretical bounds among a set of 96 realizations, consisting in various initialization. For case $(a)$ and $(b)$, the corresponding effective properties are reported in figure 13, the filled triangle correspond to case $(a)$ and the empty triangle correspond to case $(b)$.

In Figure 14, we present snapshot of the optimization process for case $(a)$. The unit cell at initialization (top left), "search" step iterations (top middle and top right), end of "search" step (top right), end of "isotropizing" step (bottom middle) and final values of optimized properties (bottom right) are reported. We first observe that the contraints on volume and bulk modulus are fullfiled, and that the final CTE is negative and close from the theoretical one. The final isotropy error is also smaller than $1 \%$.

Result for case $(b)$ is illustrated similarly in figure 15. For this case, the designed microstructure results in properties closer to the theoretical bound. The error estimator is slightly larger thant for case (a) but isotropy is found reasonably. The constraint in volume fraction of phase 4 is not completely fullfilled ( 0.23 attained while 0.25 is prescribed) but acceptable.

In Figure 14 and 15, we observe that the proposed design employs a bending mechanism to control thermal expansion like the one shown in Figure $1 \mathrm{~b}$ ).

\subsection{Non-monotonic interface profile}

In this section results that account for the effect of graded and non-monotonic interfaces are presented. The mechanical properties of the constitutive phases are these reported in table 1 . We now 


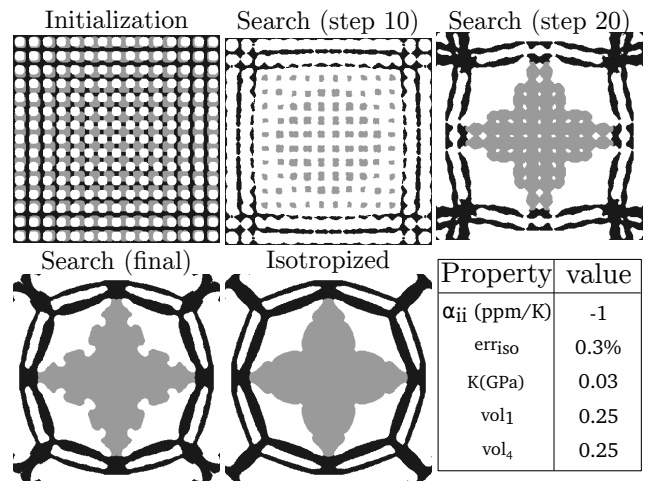

Figure 14: Optimization Case (a) (less stiff modulus constraint), with compressibility modulus, $K>0.03$, and monotonic interface transitions.

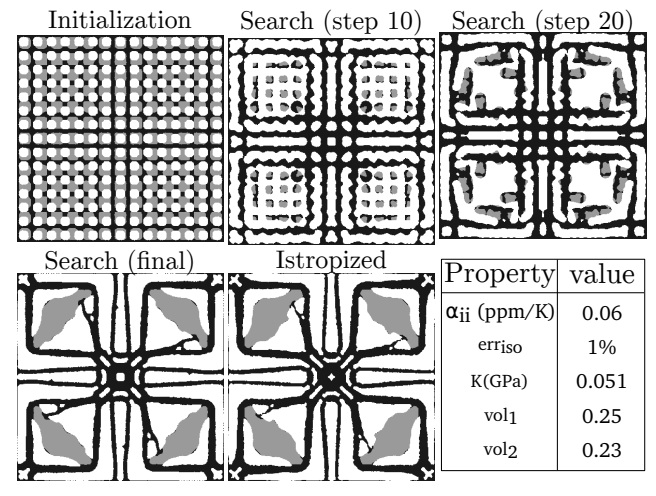

Figure 15: Optimization Case (b) (stiffer modulus constraint), with compressibility modulus, $K>0.05$, and monotonic interface transitions.

consider a non-monotonic transition between the constitutive phases as depicted in Figure 16. First, we present the results with the interface region that has a stiffer response than the surrounding bulks which have identical Young's modulus and Poisson's ratio, their CTE being different by a factor of 10. We observe in figure 13 that accounting for a stiffer interface results in a lower CTE as the optimized microstructure presented in Figure 14. In contrast, Figure 17 presents a simulation where interface is more compliant thant the bulk materials, for which the effective properties are also reported in Figure 13

Figures 16 and 17 are results for the compliant effective bulk modulus constraint Case $(a)$ $(K>0.03)$. These can be compared with the results in Figure 14, and are represented by the red triangle and blue triangle, respectively, in Figure 13. As in the previous results, the constraints on the general optimization problem are fulfilled. Similarly for the Case (b), Figure 18 can be compared to Figure 15.

By comparing results in Figures 14, 16 and 17, where only the profile of the interface properties is changed, we observe that the optimal shapes are noticeably different. The performance of these three architectured materials is also different (see Figure 13). It appears that, for this optimization problem, a stiffer interface has a positive effect on the performance whereas, a less stiff interface transition is detrimental to reach the theoretical performance or extreme bound. Based on the optimized shapes, the conceptual mechanism for effective thermal expansion control differs markedly between the monotonic interface case (see Figure 14) and the stiffer interface case (see Figure 16). In the monotonic interface case (Figure 14) where the interface properties are a weighted average of the bulk materials/phases, a flexion mechanism operates. In the case of a stiffer interface(Figure 16), a planar "bi-metallic" mechanism is promoted. 


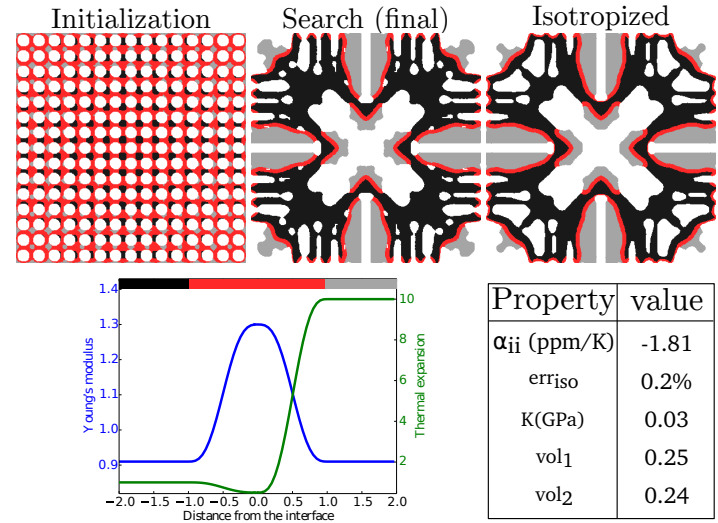

Figure 16: Optimization Case (a) (compliant effective modulus constraint), with compressibility modulus, $K>0.03$, and non-monotonic interface transitions. In this case, the nonmonotonic interface transition allows for the interfacial properties to be stiffer than either of the bulk constituent materials. The interfacial zone is highlighted in red.

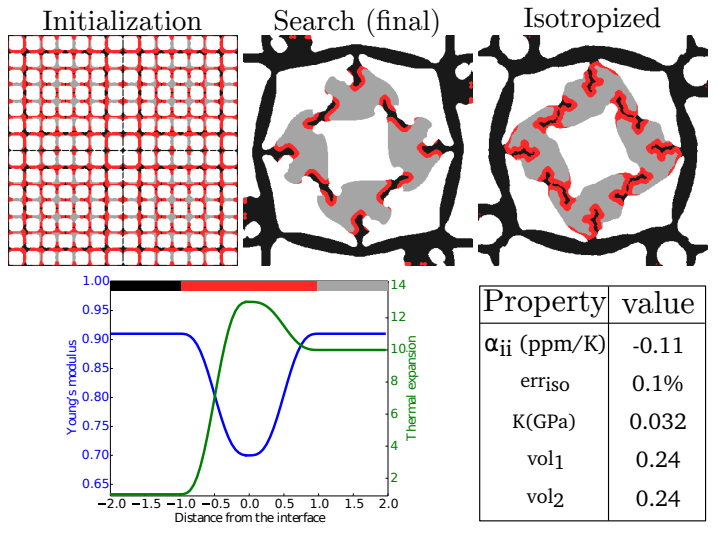

Figure 17: Optimization Case (a) (compliant effective modulus constraint), with compressibility modulus, $K>0.03$, and non-monotonic interface transitions. In this case, the nonmonotonic interface transition(see above profile) allows for the interfacial mechanical properties to be weaker than either of the bulk constituent materials. The interfacial zone is highlighted in red.

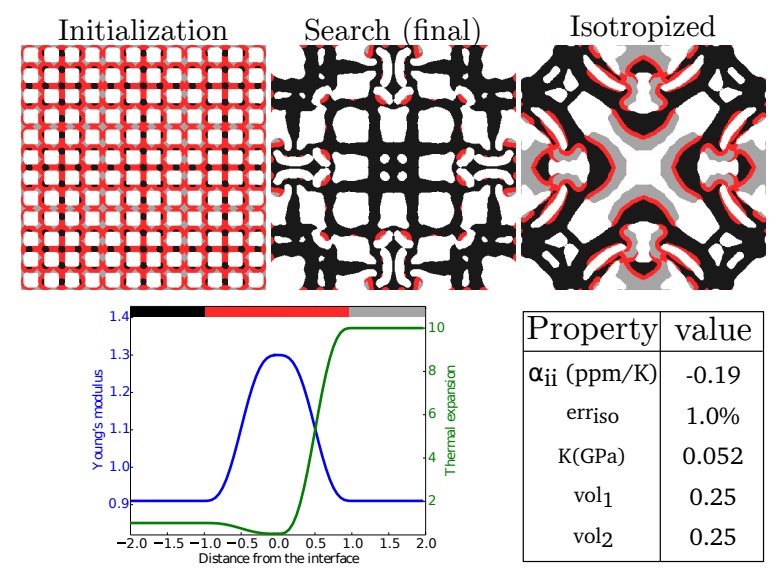

Figure 18: Optimization Case (b) (stiffer effective modulus constraint), with compressibility modulus, $K>0.05$, and non-monotonic interface transitions. In this case, the non-monotonic interface transition(see above profile) allows for the interfacial mechnaical properties to be stiffer than either of the bulk constituent materials.

\section{Discussion}

This section addresses comments on the method adopted in this study and highlights some issues and their proposed solutions. 


\subsection{On the global method}

A level set shape optimization methodology is presented to attain extreme effective thermoelastic properties for a periodic composite of which properties are estimated by periodic homogenization.The present study somehow combines approaches proposed by Wang et al. (2004) and Sigmund and Torquato (1997). Wang et al. (2004) does not ensure isotropic properties and therefore are not comparable to the theoretical bounds established by Gibiansky and Torquato (1997). In Sigmund and Torquato (1997), topology optimization is used to address the problem under consideration here but the influence of graded interfaces is not explored. Thus, the present work extends these contribution by accounting for specific interface properties as proposed by Vermaak et al. (2013). We evidence that considering different interface properties can influence the mechanism to attain target effective thermal expansion. This is an important point because it implies that an optimal design obtained without taking interfaces into account may not be optimal if interface effects are not negligible.

It is worth noting that our methodology employs an automated and systematic optimimization method that does not require human interaction during the optimization process. In contrast, and as mentioned in Sigmund and Torquato (1997), the SIMP method that produced the results therein reported is less automated and does require human interaction: "To arrive at an optimal solution, depending on the initial guess, several thousand iterations are needed. Including interaction by the user [...]" Sigmund and Torquato (1997)(sec 2.4, page 1049).

As it is common in shape optimization problems, the method reported here is sensitive to the initialization step. Results presented here are the best results from a series of 96 realization with different initial domains. The initializations employ a square shaped pattern of inclusions, it seems to lead to better optimized shapes than the initialization with circular inclusions commonly used in the literature. We only change the number of holes for both of the two level sets, from $12 \times 12$ holes to $25 \times 25$. The motivation for defining such range is that shape optimization is not able to create holes (except in some restrictive cases), so the initial number of holes globaly determines the complexity of the final shape. On the other hand, the number of holes has to be small enough such that their spacial period stays larger than the element size of the finite element mesh. Figure 19 presents microstructure designs that are from the same series of simulations that lead to the result presented in Figure 14. All of these structures fulfill the prescribed constraints, but all of them also have a higher effective CTE as that of Figure 14: each of these alternate architectures corresponds to a local minimum of the optimization problem. The suggestion is that, by exploring more initial configuration or by including user-interaction during the optimization procedure, designs that coincide or get close to the theoretical property bounds could be found.

\subsection{On the control of interfaces}

As presented in Vermaak et al. (2013), accounting for graded interfaces may induce some numerical artefacts, depending on the prescribed interface properties. This is illustrated in Figure 20. In more general terms, in the case where the graded properties of the interface are beneficial to fulfill the problem's objective, the optimization process will promote as much interface as possible resulting in a tortuous design. This is a logical trend, but it should be controlled in order to promote manufacturable designs. In his study, stiffer interfaces are beneficial to the optimization problem as they bring a higher stiffness. Thus if they are not controlled, the algorithm takes advantage of it and build interface network (see Figure 20). This is due to a phenomenon that we call the "ghost link effect", depicted in Figure 21. We observe two parts of the domain $\Omega_{1}$ separated by a small 

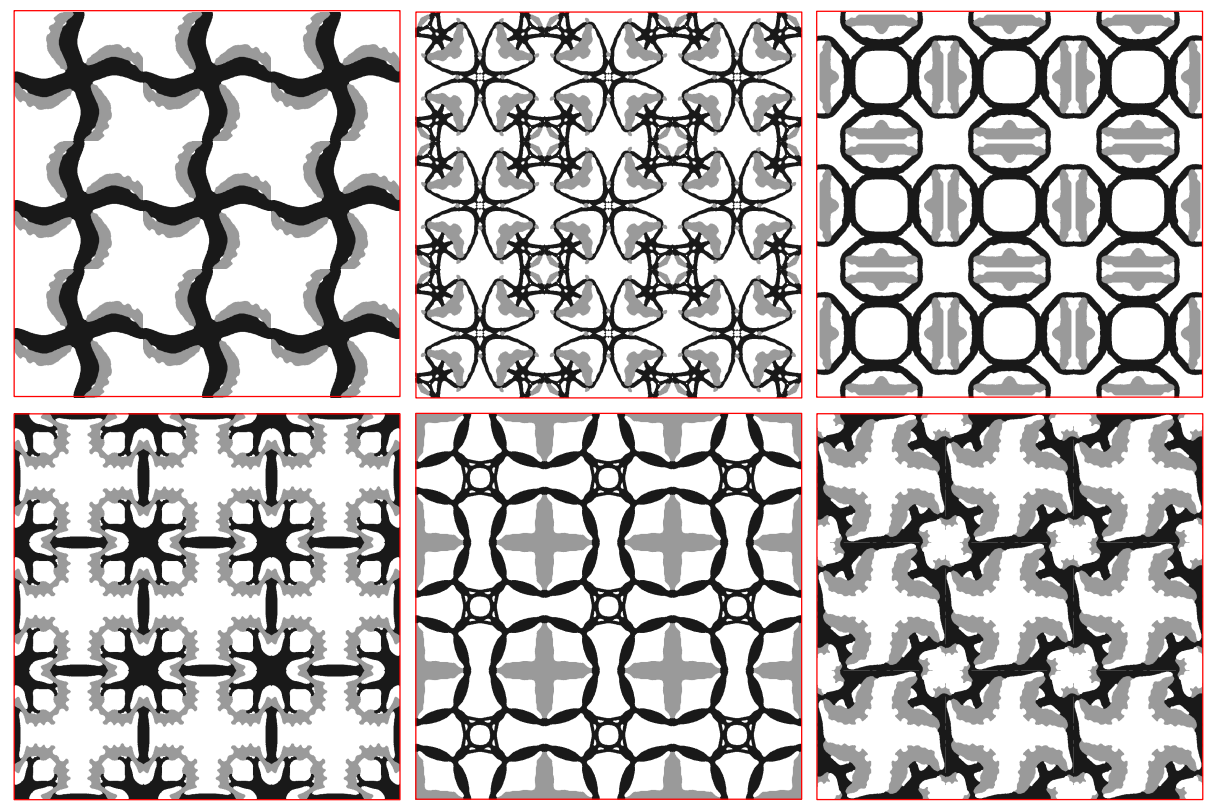

Figure 19: Examples of less optimal architectures obtained with optimization Case (a) (less stiff effective compressibility modulus constraint).

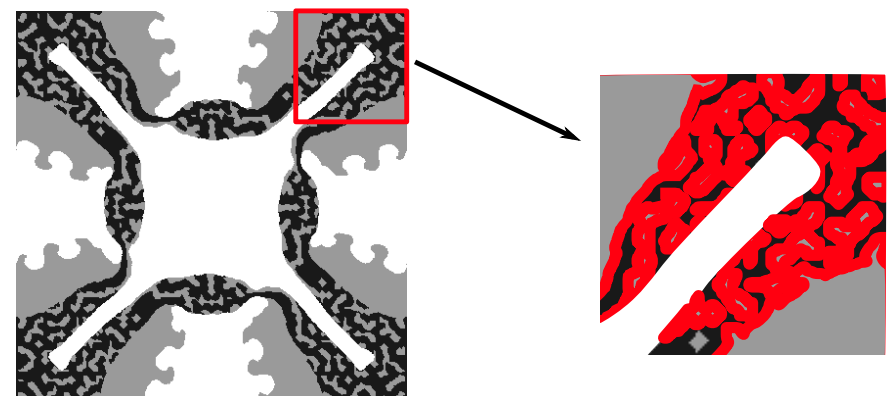

Figure 20: Illustration of the distributed interface network effect. In this non-monotonic graded interface example, the interface between the gray and black bulk materials has stiffer properties than the bulk materials. On the left: The distribution of phases in the final design creates regions that are dominated by an interface network. On the right: A magnified zone of the solution on the left. Here the interface are highlighted in red to show how the optimizer promotes the formation of small inclusions in order to form a distributed network of interfaces.

strip of domain $\Omega_{2}$. The variation of properties when crossing the interface between $\Omega_{1}$ and $\Omega_{2}$ is depicted at the top-right, it is a case whith a stiffer interface. The transition $\Omega_{1} \rightarrow \Omega_{2} \rightarrow \Omega_{1}$ shows a numerical artefact: even if the two parts of domain $\Omega_{1}$ are supposed to be mecanicaly disconnected, they are connected by a "ghost link" due to the interpolation scheme.

The Figure 22 then shows how this effect can be used to generates a high stiffness area, which 

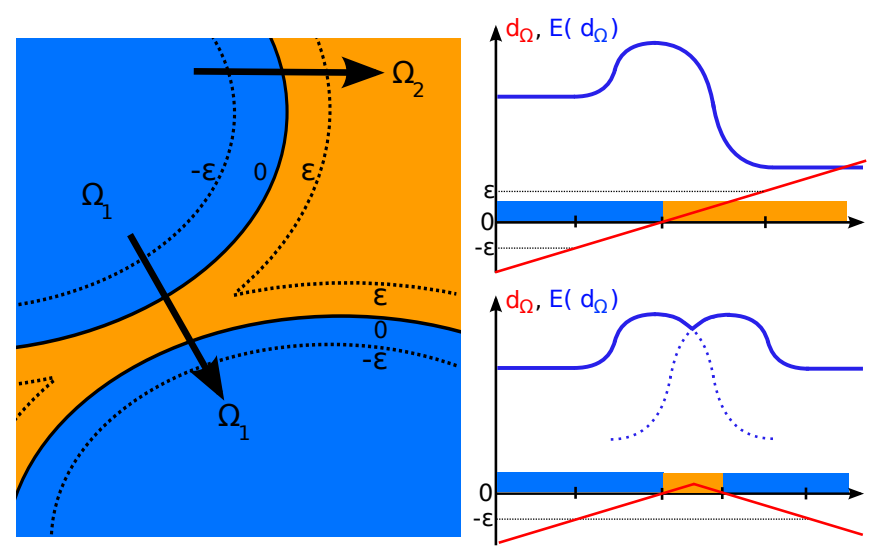

Figure 21: Feature of the graded interface formulation. Sometimes two distinct regions of a material, $\Omega_{1}$, that are ostensibly separated by a region of another material, $\Omega_{2}$, can encroach upon eachother, replacing the $\Omega_{2}$ region altogether. As a result, regions of material $\Omega_{1}$ are connected purely by "interface" and without any of the other $\Omega_{2}$ material. Depending on the interpolation scheme used, this can create an artificial stiff link between regions of the same material as illustrated here.

is not physically acceptable.
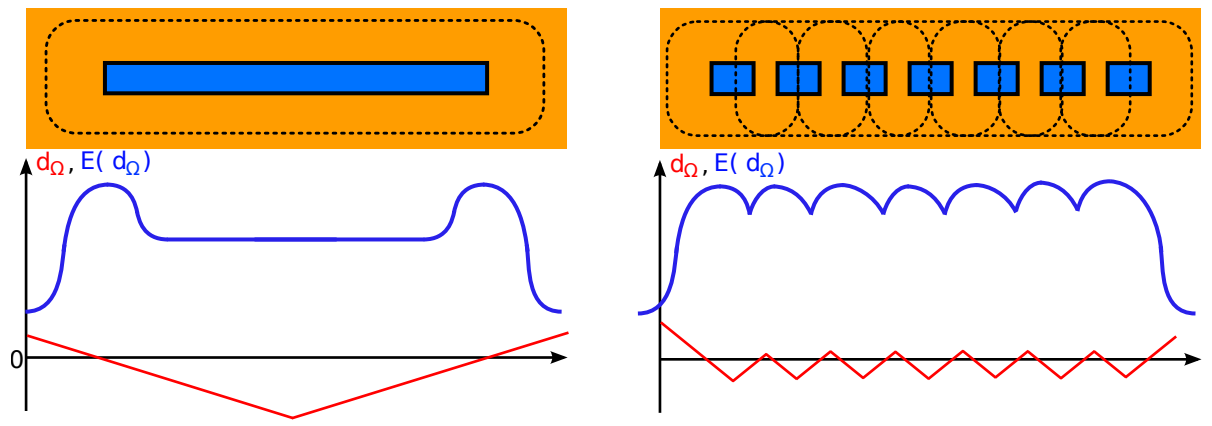

Figure 22: Use of the ghost link effect to generate a high stiffness rod.

To avoid this type of behavior, we proposed to use a simple method that suppress ghost link effect, and so all the directly induced defects. This is a simple shifting of the level set function that move the variation of properties inside the solid phase, this is a simple transformation of equation 21:

$$
\begin{gathered}
C_{i j k l}\left(d_{\Omega}+d_{0}\right)= \\
\left\{\begin{array}{cl}
C_{i j k l}^{1}+\left(C_{i j k l}^{i n t}(\theta)-C_{i j k l}^{1}\right) \cdot h_{m}\left(d_{\Omega}+d_{0}\right) & x \in \Omega \\
C_{i j k l}^{i n t}(\theta)+\left(C_{i j k l}^{v o i d}-C_{i j k l}^{i n t}(\theta)\right) \cdot h_{p}\left(d_{\Omega}+d_{0}\right) & x \in D \backslash \Omega
\end{array}\right.
\end{gathered}
$$

Where $d_{0}$ is the shifting parameter.

As illustrated by figure 23, adding a shifting parameter avoid ghost link effect. Mathematically 
speaking, $d_{0}$ could be defined as a projection, but this parameter also has more physical sense, depending on its value:

- If $d_{0}<0$ : It is the minimum thickness of a piece of matter.

- If $d_{0}>0$ : It is the minimum thickness of a piece of void.

A convenient value for $d_{0}$ is $\epsilon$, so all the graded interface is moved in the negative part of the level set.
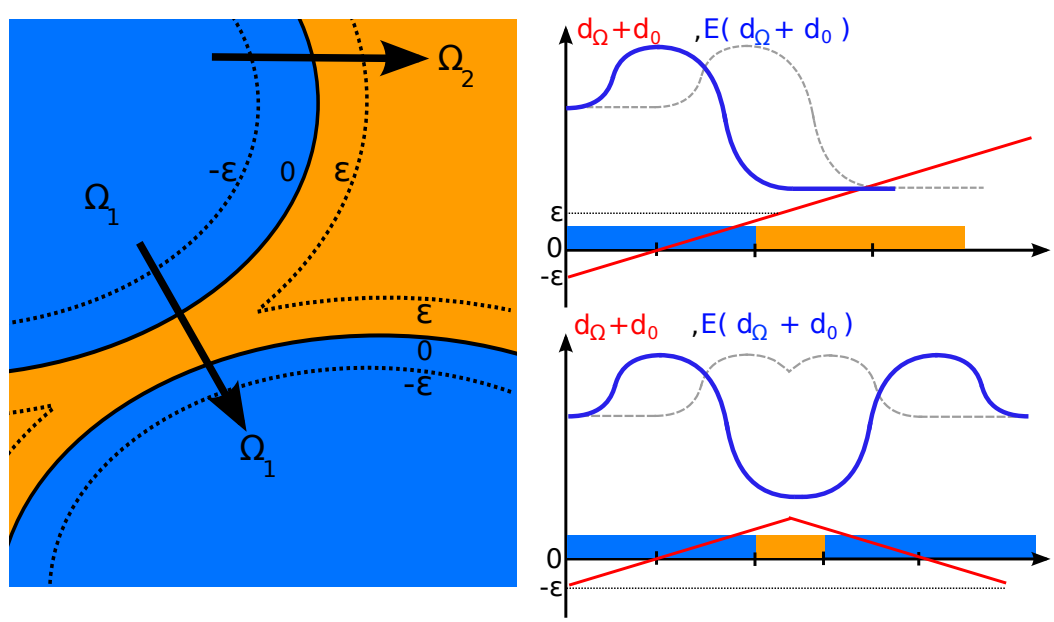

Figure 23: Shift of the interpolation scheme in order to avoid the ghost link effect.

\section{Conclusion}

We have investigated the effect of graded interfaces on the optimized performance and predicted topology of thermoelastic composites. Engineering interfaces, whether chemically or geometrically, is the frontier for accessing new and extremal functionalities and properties in composite design. To this end, we have used a level-set approach that takes advantage of recent developments (Vermaak et al., 2013; Allaire et al., 2014) for the treatment of interfaces. Depending on whether or not interfacial properties are beneficial to the objective function, the optimization algorithm may try to build a distributed network of interfaces. When this is not desired, a projection scheme is suggested to control the interface behavior. When incorporating interface effects, the framework for designing the optimization procedures must change. Carefully considering the interfacial interactions between different materials/phases or higher order combinations of materials/phases is a crucial step. Regardless of the prescribed interfacial properties, more approaches to model, handle, and harness the transition regions between bulk materials or phases are needed. Lastly, recent developments in manufacturing methods are making directly printing multi-material or multi-phase composites more and more feasible. This offers new possibilities for realizing extremal architectured materials that shape and topology optimizations identify. This study shows that including interface behavior in transition zones between materials and phases is important because it not only changes optimized performance, but also optimized shapes. 
In this study we proposed a shape optimization method applied for the design of periodic heterogeneous materials with explicit account for graded interfaces. A numerical modeling based on Vermaak et al. (2013) is presented and the shape derivation of quantities that depend on a particular interpolation scheme has been presented. The final framework is then used for the optimization of microstructures of which properties meet extreme theoretical bound proposed by Gibiansky and Torquato (1997). By considering different profiles for the interface properties and also different cases, we evidence that the interfaces govern the local mechanism required to meet the overall effective properties and moreover the final design of optimized microstructures. This is thought to be an important issue when manufacturing such architectured materials. In addition, a method that prevent numerical artefact in the interface distribution is reported.

\section{References}

Allaire, G. (2002). Shape optimization by the homogenization method, volume 146 of Applied Mathematical Sciences. Springer-Verlag, New York.

Allaire, G. (2007). Conception optimale de structures, volume 58 of Mathématiques Es Applications (Berlin). Springer-Verlag, Berlin.

Allaire, G., Dapogny, C., Delgado, G., and Michailidis, G. (2014). Mutli-phase structural optimization via a level-set method. ESAIM: Control, Optimisation and Calculus of Variations, 20, pp 576-611. doi:10.1051/cocv/2013076.

Allaire, G., Jouve, F., and Toader, A.-M. (2004). Structural optimization using sensitivity analysis and a level-set method. Journal of computational physics, 194(1):363-393.

Andreassen, E., Lazarov, B., and Sigmund, O. (2014). Design of manufacturable 3d extremal elastic microstructure. Mechanics of Materials, 69(1):1-10.

Ashby, M. (2005). Hybrids to fill holes in material property space. Philosophical Magazine, 85(26$27): 3235-3257$.

Ashby, M. (2013). Designing architectured materials. Scripta Materialia, 68(1):4-7.

Ashby, M. and Brechet, Y. (2003). Designing hybrid materials. Acta materialia, 51(19):5801-5821.

Bendsøe, M. and Kikuchi, N. (1988). Generating optimal topologies in structural design using a homogenization method. Computer methods in applied mechanics and engineering, 71(2):197224 .

Brechet, Y. and Embury, J. (2013). Architectured materials: Expanding materials space. Scripta Materialia, 68(1):1-3.

Céa, J. (1986). Conception optimale ou identification de formes: calcul rapide de la dérivée directionnelle de la fonction coût. Modélisation mathématique et analyse numérique, 20(3):371-402.

Compton, B. and Lewis, J. (2014). 3d-printing of lightweight cellular composites. Advanced Materials, 26(34):5930-5935. 
Creton, C., Kramer, E., Brown, H., and Hui, C. (2001). Adhesion and Fracture of Interfaces Between Immiscible Polymers : from the Molecular to the Continuum Scale. Polymer, 156(0001):53-136.

Gibiansky, L. and Torquato, S. (1997). Thermal expansion of isotropic multiphase composites and polycrystals. Journal of the Mechanics and Physics of Solids, 45(7):1223-1252.

Jefferson, G., Parthasarathy, T., and Kerans, R. (2009). Tailorable thermal expansion hybrid structures. International Journal of Solids and Structures, 46(11):2372-2387.

Kieback, B., Neubrand, A., and Riedel, H. (2003). Processing techniques for functionally graded materials. Materials Science and Engineering: A, 362(1):81-106.

Markworth, A., Ramesh, K., and Parks, W. (1995). Modelling studies applied to functionally graded materials. Journal of Materials Science, 30(9):2183-2193.

Mines, R., Tsopanos, S., Shen, Y., Hasan, R., and McKown, S. (2013). Drop weight impact behaviour of sandwich panels with metallic micro lattice cores. International Journal of Impact Engineering, 60:120-132.

Osher, S. and Sethian, J. (1988). Fronts propagating with curvature-dependent speed: algorithms based on hamilton-jacobi formulations. Journal of computational physics, 79(1):12-49.

Sethian, J. (1999). Level set methods and fast marching methods: evolving interfaces in computational geometry, fluid mechanics, computer vision, and materials science. Cambridge university press.

Sethian, J. and Wiegmann, A. (2000). Structural boundary design via level set and immersed interface methods. Journal of computational physics, 163(2):489-528.

Sigmund, O. (2000). A new class of extremal composites. Journal of the Mechanics and Physics of Solids, 48(2):397-428.

Sigmund, O. and Torquato, S. (1997). Design of materials with extreme thermal expansion using a three-phase topology optimization method. Journal of the Mechanics and Physics of Solids, 45(6):1037-1067.

Suard, M., Lhuissier, P., Dendievel, R., Blandin, J.-J., Vignat, F., and Villeneuve, F. (2014). Towards stiffness prediction of cellular structures made by electron beam melting (ebm). Powder Metallurgy, 57(3):190-195.

Tan, C., Wang, G., Ji, L., Tong, Y., and Duan, X.-M. (2016). Investigation on 316L/W functionally graded materials fabricated by mechanical alloying and spark plasma sintering. Journal of Nuclear Materials, 469:32-38.

Torquato, S. (2010). Optimal design of heterogeneous materials. Annual Review of Materials Research, 40:101-129.

Vermaak, N., Michailidis, G., Faure, A., Parry, G., Estevez, R., Jouve, F., Allaire, G., and Bréchet, Y. (2016). Topological optimization with interfaces. to appear in: Archimats: Architectured Materials in Nature and Engineering, Springer. 
Vermaak, N., Michailidis, G., Parry, G., Estevez, R., Brechet, Y., and Allaire, G. (2013). Material interface effects on the topology optimization of multi-phase thermoelastic structures using a level set method. (submitted in SMO: Structural and Multi-disciplinary Optimization).

Wang, M., Wang, X., and Guo, D. (2003). A level set method for structural topology optimization. Computer methods in applied mechanics and engineering, 192(1):227-246.

Wang, X., Mei, Y., and Wang, M. (2004). Level-set method for design of multi-phase elastic and thermoelastic materials. International Journal of Mechanics and Materials in Design, 1(3):213239 . 